Pacific Journal of Mathematics

MONOTONE DECOMPOSITIONS INTO TREES OF
HAUSDORFF CONTINUA IRREDUCIBLE ABOUT A FINITe
SUBSET

ELDON JON Nought 


\title{
MONOTONE DECOMPOSITIONS INTO TREES OF HAUSDORFF CONTINUA IRREDUCIBLE ABOUT A FINITE SUBSET
}

\author{
ELdon J. Vought
}

\begin{abstract}
This paper deals with characterizing two types of monotone, upper semi-continuous decompositions of a Hausdorff continuum that is irreducible about a finite subset. One of the decompositions is minimal with respect to the property of having a quotient space which is a tree (a hereditarily unicoherent, locally connected continuum) and is characterized in terms of certain collections of subcontinua. The other decomposition is not only minimal but also unique with respect to the properties that the quotient space is a tree and the elements of the decomposition have void interiors. This decomposition is characterized quite simply by prohibiting the existence of indecomposable subcontinua with nonvoid interiors. The structure of the elements of the decompositions that have void interiors is very nice and is described by means of the aposyndetic set function $T$. In the case where elements exist with nonvoid interiors, the structure can be very complicated and a final result deals with this structure under some rather stringent conditions.
\end{abstract}

For a compact, metric continuum $M$ that is irreducible about two points Thomas proved [7, p. 15] that $M$ has a decomposition $\mathscr{D}$ such that

(1) $\mathscr{D}$ is upper semi-continuous,

(2) the elements of $\mathscr{D}$ are continua,

(3) each element of $\mathscr{D}$ has void interior, and

(4) the quotient space of $\mathscr{D}$ is an arc,

if and only if $M$ contains no indecomposable subcontinuum with nonvoid interior. Gordh [3, p. 650] generalized this for a compact, Hausdorff continuum where the quotient space in his result is a generalized arc (a continuum in which every point except for two is a separating point). Theorem 1 in this paper generalizes the above decomposition theorem to a compact, Hausdorff continuum that is irreducible about a finite set of points where condition (4) is now that the quotient space is a tree (a locally connected, hereditarily unicoherent continuum). This result strengthens somewhat a theorem due to Russell [6, p. 260] which proves that a sufficient condition in order for a metric continuum $M$ to have such a decomposition is that $M$ be hereditarily decomposable. 
If $M$ is a compact, Hausdorff continuum that is irreducible about a finite set of points then in Theorem 2 necessary and sufficient conditions are given so that $M$ has a nontrivial decomposition $\mathscr{D}$ for which

(1) $\mathscr{D}$ is upper semi-continuous,

(2) the elements of $\mathscr{D}$ are continua, and

(3) the quotient space is a tree.

These results generalize and strengthen a theorem of the author's [8] for a continuum that is irreducible about two points.

The difference in Theorems 1 and 2 is that the elements of the decomposition must have void interiors in Theorem 1 . The elements in this decomposition have a very nice structure indeed and, in fact, the decomposition is unique. The decomposition in Theorem 2 is not unique with respect to properties (1), (2), (3), but is minimal in the sense of refinement and enjoying these properties. In this decomposition the element structure is quite complicated. A final theorem of this paper allows $M$ to contain certain combinations of indecomposable subcontinua with nonvoid interiors and a decomposition results that is very similar to that of Theorem 1 .

Some terminology and a few notions are necessary in order to prove the first theorem. Let $M$ be a compact, Hausdorff continuum and $A$ a subset of $M$. Then $T(A)$ is the set $A$ together with all points $x \in M$ for which there does not exist an open set $U$ and continuum $H$ such that $x \in U \subset H \subset M$ - $A$. If $n=1$ let $T^{n}(A)=T(A)$ and if $n \geq 2$ let $T^{n}(A)=T\left(T^{n-1}(A)\right)$. If $T(A)=A$ let us say that $A$ is $T$-closed and if $T(x)=\{x\}$ for each $x \in$ $M$ we say that $M$ is semi-locally connected. The set function $T$ is the aposyndetic set function first defined by Jones [5]. For more information on $T$ and $T^{n}$ see [1]. If $M$ is irreducible about the $n$ points $x_{1}, x_{2}, \ldots, x_{n}$ but is not irreducible about any fewer number of points, then $M$ is minimally irreducible about $x_{1}, x_{2}, \ldots, x_{n}$ and each $x_{i}, 1 \leq i \leq n$, is an end point of $M$. A decomposition $\mathscr{D}$ of $M$ that has a specific set of properties is a core decomposition with respect to these properties if $\mathscr{D}$ refines every other decomposition of $M$ having these properties. FitzGerald and Swingle have proved that if $M$ is a compact, Hausdorff continuum, then $M$ has a core decomposition with respect to the properties of being monotone and having a semi-locally connected quotient space [2, p. 37]. We will use this result.

THEOREM 1. Let $M$ be a compact, Hausdorff continuum that is minimally irreducible about $n$ points, $n \geq 2$. If $M$ contains no indecomposable subcontinuum with nonvoid interior then there exists a decomposition $\mathscr{D}$ of $M$ such that

(1) $\mathscr{D}$ is upper semi-continuous, 
(2) the elements of $\mathscr{D}$ are continua,

(3) the quotient space of $\mathscr{D}$ is locally connected, and

(4) each element of $\mathscr{D}$ has void interior.

Furthermore, the quotient space is a tree minimally irreducible about $n$ points and $\mathscr{D}$ is the only decomposition satisfying (1), (2), (3), (4). Conversely, if $\mathscr{D}$ is a decomposition of $M$ satisfying (1), (2), (3), (4) then $M$ contains no indecomposable subcontinuum with nonvoid interior.

Proof. Let each indecomposable subcontinuum of $M$ have void interior. We will first show that $T^{n}(x)=T^{n+1}(x)$ for all $x \in M$ and that $\left\{T^{n}(x) \mid x \in M\right\}$ is a decomposition of $M$. Then by [2, p. 39], $\left\{T^{n}(x), x \in\right.$ $M$ ) will be the core decomposition of $M$ with respect to being monotone, upper semi-continuous and having a semi-locally connected quotient space. Given $x \in M$ clearly $T^{n}(x) \subset T^{n+1}(x)$. To show $T^{n+1}(x) \subset T^{n}(x)$ suppose $y \notin T^{n}(x)$. There exists a continuum $H_{1}$ such that $y \in H_{1}^{o} \subset H_{1} \subset$ $M-T^{n-1}(x)$. The components of $M-H_{1}$ are open sets since there can be only a finite number due to the fact that $M$ is irreducible about a finite set. So $H_{1}$ can be chosen so that $M-H_{1}$ is connected. Let $K_{1}={\overline{M-H_{1}}}_{1}$. For each $z \in H_{1}$ there is a continuum $H_{z}$ such that $z \in H_{z}^{o} \subset H_{z} \subset M-$ $T^{n-2}(x)$ and hence, by the compactness of the continuum $H_{1}$, there exists a continuum $H_{2}$ such that $H_{1} \subset H_{2}^{o} \subset H_{2} \subset M-T^{n-2}(x)$. Again we may assume that $\mathrm{M}-\mathrm{H}_{2}$ is connected and we set $\mathrm{K}_{2}=\mathrm{M}-\overline{\mathrm{H}}_{2}$. Continuing this process we obtain $n$ continua $H_{1}, H_{2}, \ldots, H_{n}$ for which the following sequence of inclusions holds:

$$
y \in H_{1}^{o} \subset H_{1} \subset H_{2}^{o} \subset H_{2} \subset \cdots \subset H_{n}^{o} \subset H_{n} \subset M-\{x \mid .
$$

Let $K_{i}=\overline{M-H}_{i}, i=1, \ldots, n$, where $M-H_{i}$ is connected. Consider the pairwise disjoint open sets

$$
H_{1}^{o}, H_{2}^{o} \cap K_{1}^{o}, H_{3}^{o} \cap K_{2}^{o}, \ldots, H_{n m-m+1}^{o} \cap K_{n m-m}^{o}, K_{n m-m+1}^{o} \text {. }
$$

Since $K_{1}$ and $H_{n}$ are continua and $M$ is irreducible about $x_{1}, x_{2}, \ldots, x_{n}, H_{1}^{o}$ and $K_{n}^{o}$ must each contain an end point of $M$. Because $M$ has but $n$ end points, one of the above $n+1$ open sets, say $H_{i}^{o} \cap K_{i-1}^{o}$, contains no end point of $M$. Let $L$ be an irreducible continuum from $H_{i-1}$ to $K_{i}$. Then $H_{i}^{o}$ $\cap K_{i-1}^{0} \subset L$ or else $H_{i-1} \cup L \cup K_{i}$ is a proper subcontinuum of $M$ containing all of the end points. Therefore $L^{o} \neq \varnothing$ and $L=L_{1} \cup L_{2}$ where $L_{1}$ and $L_{2}$ are proper subcontinua of $L$. Due to $L$ being irreducible we have $L_{1} \cap H_{i-1} \neq \varnothing \neq L_{2} \cap K_{i}$ and $L_{1} \cap K_{i}=\varnothing=L_{2} \cap H_{i-1}$. We now have the inclusions

$$
T(x) \subset K_{n}, T^{2}(x) \subset K_{n-1}, \ldots, T^{n-i+1}(x) \subset K_{i},
$$




$$
\begin{aligned}
T^{n-i+2}(x) \subset K_{i} \cup L_{2}, T^{n-i+3}(x) \subset K_{i-1}, \ldots, & \\
& T^{n}(x) \subset K_{2}, T^{n+1}(x) \subset K_{1} .
\end{aligned}
$$

Therefore $y \notin T_{1}^{n+1}(x)$ and it follows that $T^{n}(x)=T^{n+1}(x)$.

To show $\left\{T^{n}(x) \mid x \in M\right\}$ is a decomposition, suppose $z \in T^{n}(x)$. By the above argument it is clear that $x \in T^{n}(z)$. We then have

$$
T^{n}(z) \subset T^{n}\left(T^{n}(x)\right)=T^{2 n}(x)=T^{n}(x) \text { and } T^{n}(x) \subset T^{2 n}(z)=T^{n}(z) .
$$

Then $T^{n}(x)=T^{n}(z)$. So if $z \in T^{n}(x) \cap T^{n}(y)$ we must have $T^{n}(x)=$ $T^{n}(z)=T^{n}(y)$ and, consequently, $\left\{T^{n}(x) \mid x \in M\right\}$ is a decomposition. As mentioned, the decomposition is monotone, upper semi-continuous and the quotient space $M^{\prime}$ is semi-locally connected. Since $M$ is minimally irreducible about $n$ points so is $M^{\prime}$, and it follows easily from this and the semi-local connectedness that $M^{\prime}$ is locally connected.

The continuum $M^{\prime}$ is a tree if and only if given any two distinct points $x$ and $y$ of $M^{\prime}$ there is a point $z \in M^{\prime}$ such that $z$ separates $x$ from $y$ [9]. Take $x, y \in M^{\prime}$ and let $H$ be an irreducible subcontinuum of $M^{\prime}$ from $x$ to $y$. Choose $z \in H$ such that $z \notin\left\{x, y, f\left(x_{1}\right), f\left(x_{2}\right), \ldots, f\left(x_{n}\right)\right\}$ where $f$ is the quotient map from $M$ onto $M^{\prime}$. The components of $M^{\prime}-\{z\}$ are open sets since $M^{\prime}$ is locally connected. Suppose $x$ and $y$ are in the same component $C$ of $M^{\prime}-\{z\}$. Let $U$ be an open set such that $z \in U, x, y \notin U$, and $f\left(x_{i}\right) \notin U$, $i=1, \ldots, n$. By the local connectedness of $M^{\prime}$ and because $z$ does not separate $\bar{C}=C \cup\{\}$, there exists a continuum $K$ such that $C-U \subset K^{o} \subset$ $K \subset C$. The open set $C-K \subset H$ for otherwise $\left(M^{\prime}-C\right) \cup K \cup H$ is a proper subcontinuum of $M^{\prime}$ containing $f\left(x_{i}\right), i=1, \ldots, n$. Choose $w \in C$ - K. If $w$ separates $z$ from $K$ in $\bar{C}$ then $w$ separates $\{x, y\}$ from $z$ in $H$ which means that $H$ is not irreducible from $x$ to $y$. So $w$ does not separate $z$ from $K$ in $\bar{C}$ and now, because $M^{\prime}$ is locally connected, there exists a subcontinuum $L$ of $\bar{C}-\{w\}$ such that $z \in L$ and $L \cap K \neq \varnothing$. But $\left(M^{\prime}-C\right) \cup L \cup K$ is a proper subcontinuum of $M^{\prime}$ containing $f\left(x_{i}\right), i=1, \ldots, n$, a contradiction. So the assumption that $x$ and $y$ are in the same component of $M^{\prime}-$ $\{z\}$ is contradictory. Hence $x$ and $y$ are in different components of $M^{\prime}-$ $\{z\}$ which means that $z$ separates $x$ from $y$ in $M^{\prime}$.

To show (4) suppose for some $x \in M$ that $T^{n}(x)$ has nonempty interior. First suppose $T^{n}(x)$ contains none of the end points. Let $K_{1}$, $K_{2}, \ldots, K_{m}$ be the closures of the finite number of components of $M-$ $T^{n}(x)$ and let $Q$ be a subcontinuum of $T^{n}(x)$ irreducible about the closure of $\left(T^{n}(x)\right)^{\circ}$. Now $Q$ is irreducible about a finite set of points, is not indecomposable and contains no indecomposable subcontinuum with 
nonvoid interior. Therefore there exist two points $x^{\prime}, y^{\prime}$ such that $x^{\prime} \in K_{i} \cap$ $Q, y^{\prime} \in K_{j} \cap Q$ for some pair of integers $i, j ; 1 \leq i, j \leq m$; and for which the following inclusions hold:

$$
y^{\prime} \in Q_{1}^{o} \subset Q_{1} \subset Q_{2}^{o} \subset Q_{2} \subset \cdots \subset Q_{n}^{o} \subset Q_{n} \subset M-\left\{x^{\prime}\right\}
$$

where each $Q_{i}$ is a continuum. But then $y^{\prime} \notin T^{n}\left(x^{\prime}\right)=T^{n}(x)$, a contradiction. If $T^{n}(x)$ contains one of the end points $x_{i}$ from $\left\{x_{1}, x_{2}, \ldots, x_{n}\right\}$, then let $L$ be a subcontinuum of $M$ irreducible about $\left\{x_{1}, x_{2}, \ldots, x_{i-1}\right.$, $\left.x_{i+1}, \ldots, x_{n}\right\}$ and let $L^{\prime}$ be an irreducible subcontinuum of $M$ from $x_{i}$ to $L$. Since $L^{\prime}$ contains no indecomposable subcontinuum with nonvoid interior it has a monotone decomposition $\mathscr{D}^{\prime}$, the elements of which have void interiors, and for which the quotient space is a generalized arc [3, p. 650]. Let $D_{x_{i}}$ be the element of $\mathscr{D}^{\prime}$ that contains $x_{i}$. Clearly $T^{n}(x) \subset D_{x_{i}}$ since $x_{i}$ $\in T^{n}(x)$ and this is a contradiction to $\left(T^{n}(x)\right)^{\circ} \neq \varnothing$.

To complete the proof of sufficiency we need to show that $\left\{T^{n}(x) \mid x\right.$ $\in M\}$ is the only decomposition satisfying (1), (2), (3), (4). Let $\mathscr{P}$ be any other decomposition satisfying these properties. Since $\left\{T^{n}(x) \mid x \in M\right\}$ refines $\mathscr{H}$, there exists $h \in \mathscr{P}$ and $T^{n}(x)$ for some $x \in M$ such that $T^{n}(x) \quad h$. Let $y \in h-T^{n}(x)$. Borrowing the construction from the first part of the proof we have the continua $H_{1}, H_{2}, \ldots, H_{n} ; K_{1}, K_{2}, \ldots, K_{n}$ where $y \in H_{1}$ and $x \in K_{n}$. For some $i, H_{i}^{o} \cap K_{i-1}^{o} \subset L$ where $L$ is the irreducible subcontinuum of $M$ from $H_{i-1}$ to $K_{i}$. Clearly $L$ separates $M$ into the open sets $H_{i-1}^{o}$ and $K_{i}^{o}$ with $y \in H_{i-1}^{o}$ and $x \in K_{i}^{o}$. But $h$ contains both $x$ and $y$ so $h$ must contain $L$. Because $L$ contains an open set and $h$ cannot contain such a set we have arrived at a contradiction.

Suppose $\mathscr{D}$ is any decomposition of $M$ satisfying (1), (2), (3), (4) and let $I$ be an indecomposable subcontinuum of $M$ such that $I^{\circ} \neq \phi$. First suppose $I$ contains an end point $x_{i}$. Let $L$ be a subcontinuum of $M$ irreducible about $\left\{x_{1}, x_{2}, \ldots, x_{i-1}, x_{i+1}, \ldots, x_{n}\right\}$ and let $L^{\prime}$ be an irreducible subcontinuum of $M$ from $x_{i}$ to $L$. If $D_{x_{i}}$ is the element of $\mathscr{D}$ containing $x_{i}$ then it is clear that $D_{x_{i}} \cap L=\varnothing$. Let us denote by $f$ the quotient map of $M$ onto the locally connected quotient space $M^{\prime}$ of the decomposition $\mathscr{D}$. Because of the local connectedness of $M^{\prime}, f\left(L^{\prime}\right) \cap f(L)$ is a single element $x \in M^{\prime}$ and $f\left(L^{\prime}\right)$ is a generalized arc. So $L^{\prime}$ contains no indecomposable subcontinuum with nonvoid interior. Thus $L^{\prime} \varsubsetneqq I$. But it is clear then that for some element $D \in \mathscr{D}$ that lies in $L^{\prime}$, the continuum $I$ is separated by $D$, a contradiction.

Next suppose $I$ contains no end point. Let $K_{1}, K_{2}, \ldots, K_{m}$ be the closures of the components of $M-I$. Consider $K_{1}$. There do not exist $D_{2}$, $D_{3}, \ldots, D_{m} \in \mathscr{D}$ each intersecting $K_{1}$ such that $D_{i} \cap K_{i} \neq \varnothing, i=2, \ldots, m$; 
for otherwise $\cup_{i=1}^{m} K_{i} \cup \cup_{i=2}^{m} D_{i}$ would be a proper subcontinuum of $M$ containing all the end points. A similar statement is true for each of the $m$ $K_{i}^{\prime}$ 's. Using this fact plus the local connectedness of the quotient space $M^{\prime}$ it follows easily that there exists an element $D \in \mathscr{D}$ such that $D \subset I^{\circ}$. Because $\mathscr{D}$ is upper semi-continuous there exists an open set $U$ such that $D$ $\subset U \subset I^{\circ}$ and $U$ is the union of members of $\mathscr{D}$. Now $f(D) \in f(U)$ and since $M^{\prime}$ is locally connected there is a connected open set $W \in M^{\prime}$ such that $f(D) \in W \subset \bar{W} \subset f(U)$. Then $D \subset f^{-1}(W) \subset f^{-1}(\bar{W}) \subset U$ which implies that $f^{-1}(\bar{W})$ is a proper subcontinuum of the indecomposable continuum $I$ with a nonvoid interior. This is impossible and the proof is complete.

Next we need to borrow a definition from Whyburn. A collection $\mathscr{G}$ of subsets (not necessarily disjoint) of $M$ is a saturated collection if whenever $G \in \mathscr{I}$ and $p \notin G$ there exists $G^{\prime} \in \mathscr{Y}$ such that $G^{\prime}$ separates $p$ from $G$ in $M$, i.e., $M-G^{\prime}=A \cup B$ where $p \in A, G \subset B$, and $\bar{A} \cap B=\varnothing$ $=A \cap \bar{B}[\mathbf{1 0}$, p. 45$]$. Also we call a subset $G$ of $M$ a separator if $M-G$ is not connected.

THEOREM 2. Let $M$ be a compact, Hausdorff continuum that is minimally irreducible about the $n$ points $\left\{x_{1}, x_{2}, \ldots, x_{n}\right\}$. Suppose there exists a nonvoid saturated collection of separators each element of which is a continuum. Then $M$ has a nontrivial decomposition $\mathscr{D}$ such that

(1) $\mathscr{D}$ is upper semi-continuous

(2) the elements of $\mathscr{D}$ are continua

(3) the quotient space of $\mathscr{D}$ is locally connected.

Moreover, $\mathscr{D}$ is the core decomposition with respect to these three properties and the quotient space is a tree minimally irreducible about $m$ points, $m \leq n$. Conversely, if $\mathscr{D}$ is a nontrivial decomposition satisfying (1), (2), (3), there exists a nonvoid saturated collection of separators each element of which is a continuum.

Proof. Consider the union of all saturated collections of separators where the separators are continua and let us denote this collection by $\mathscr{G}$. Clearly $\mathscr{G}$ is itself a saturated collection of separators each element of which is a continuum and is the unique maximal such collection. Let $S_{x}$ be the set of all points $y$ such that there does not exist $G \in \mathscr{I}$ which separates $x$ from $y$. Now take $G \in \mathscr{I}$ and let $M-G=A \cup B$, a separation of $M$. Choose $a \in A, b \in B, c \in G$. There exists $G^{\prime} \in \mathscr{I}$ such that $G^{\prime}$ separates $a$ from $G$ and clearly $G^{\prime} \subset A$. So $G^{\prime}$ separates $a$ from $c$ in $M$ and hence by [2, p. 49] $\mathscr{S}=\left\{S_{x} \mid x \in M\right\}$ is an upper semi-continuous decomposition of $M$ into closed sets. Now let $b$ denote the core decomposition with respect to 
the properties of being monotone and having a semi-locally connected quotient space $(M, \mathscr{b})$ and let $f$ be the quotient map from $M$ onto $(M, \mathscr{b})$. Since $(M, \mathscr{C})$ is semi-locally connected and clearly irreducible about $m$ points, $m \leq n$, it follows that $(M, \mathscr{b})$ is locally connected. As in the proof of Theorem $1,(M, \mathscr{b})$ can be shown to be a tree. So what remains to be shown is that $\mathscr{b}=\mathscr{f}$. Let $K=\left\{f^{-1}(k) \mid k\right.$ is a point of $(M, \mathscr{b})$ and $k$ separates $(M$, $b)\}$. Since $(M, \mathscr{b})$ is a tree obviously $K \neq \varnothing$ so let $f^{-1}(k) \in K, x \in M-$ $f^{-1}(k)$ and consider $f(x), k \in(M, \mathscr{b})$. Due to the fact that $(M, \mathscr{b})$ is hereditarily unicoherent there exists a unique subcontinuum of $(M, \mathscr{b}), H$, irreducible from $f(x)$ to $k$. Choose $k^{\prime} \in H-\{f(x), k\}$. Because $(M, b)$ is locally connected it follows that $k^{\prime}$ separates $f(x)$ from $k$ in $(M, b)$. Then $f^{-1}\left(k^{\prime}\right)$ separates $x$ from $f^{-1}(k)$ in $M$ and $K$ is a saturated collection of separators of $M$. By the monotonicity of $f$ each element of $K$ is a continuum. The elements of $\mathscr{b}$ are of the form $f^{-1}(k)$ where $k \in(M, \mathscr{b})$ so take $f^{-1}(k)$ $\in \mathscr{b}$ and $x \in M-f^{-1}(k)$. Exactly as above there exists $k^{\prime} \in(M, b)$ such that $f^{-1}\left(k^{\prime}\right)$ separates $x$ from $f^{-1}(k)$. Since $f^{-1}\left(k^{\prime}\right) \in K$ then $f^{-1}\left(k^{\prime}\right) \in \mathscr{G}$ (because $\mathscr{G}$ is maximal) and it follows that $\mathscr{P} \leq \mathscr{b}$.

To prove that $\mathscr{b} \leq \mathscr{P}$ let us note that FitzGerald and Swingle have proved that $\mathscr{b}$ can alternately be expressed as the core decomposition with respect to the properties of being upper semi-continuous and having $T$ closed elements $[2$, p. 37]. We have already established that $\mathscr{S}$ is upper semi-continuous and if we show that the elements of $\mathscr{P}$ are $T$-closed it will follow that $\mathscr{b} \leq \mathscr{P}$. For this purpose take $S_{x} \in \mathscr{P}$ and $y \in M-S_{x}$. There exists $G \in \mathscr{G}$ such that $M-G=A \cup B$, a separation, with $x \in A$ and $y \in$ $B$. Because $\mathscr{I}$ is a saturated collection it follows easily that $G$ can be chosen so that $S_{x} \subset A$ and $y \in B$. But then $G \cup B$ is a continuum containing $y$ in its interior and not intersecting $S_{x}$. Hence $y \notin T\left(S_{x}\right)$ so $S_{x}$ is $T$-closed and $\mathscr{f}$ is the core decomposition $\mathscr{D}$ of the theorem.

Conversely, suppose that $\mathscr{D}$ is a nontrivial decomposition satisfying conditions (1), (2), (3) in Theorem 2. Let the quotient space of $\mathscr{D}$ be denoted by $(M, \mathscr{D})$ and let $f$ be the quotient map of $M$ onto $(M, \mathscr{D})$. We know from the proof of Theorem 1 that $(M, \mathscr{D})$ is a tree. Let $K=$ $\left\{f^{-1}(k) \mid k \in(M, \mathscr{D})\right.$ and $k$ separates $\left.(M, \mathscr{D})\right\}$ and let $f^{-1}(k) \in K, y \in M$ $-f^{-1}(k)$. Because $(M, \mathscr{D})$ is hereditarily unicoherent there exists a unique continuum $H$ irreducible between $f(y)$ and $k$. As previously proved there exists $k^{\prime} \in H-\{f(y), k\}$ such that $k^{\prime}$ separates $f(y)$ from $k$ in $(M, \mathscr{D})$. Then $f^{-1}\left(k^{\prime}\right)$ separates $y$ from $f^{-1}(k)$ in $M$. So $K$ is a nonvoid saturated collection of separators of $M$ and each separator is a continuum since $\mathscr{D}$ is monotone.

The next theorem is a generalization of Theorem 1 where $M$ is allowed to contain simple chains of indecomposable subcontinua with nonvoid interiors. A collection of subsets of $M,\left\{C_{i} \mid i=1, \ldots, m\right\}$, is a simple chain if 
$C_{i} \cap C_{j} \neq \phi$ if and only if $|i-j| \leq 1$. Each subset $C_{i}$ is called a link of the chain.

THEOREM 3. Let $M$ be a compact, Hausdorff continuum minimally irreducible about $n$ points, $n \geq 2$. If $M$ contains no simple chain of $m$ links, $m$ $\geq 1$, in which each link is an indecomposable continuum with nonvoid interior then $\mathscr{D}=\left\{T^{n m-m+1}(x) \mid x \in M\right\}$ is the core decomposition of $M$ with respect to being monotone, upper semi-continuous with a locally connected quotient space. Moreover, the quotient space is a tree minimally irreducible about $p$ points where $p \leq n$.

Proof. It is only necessary to show that $T^{n m-m+2}(x)=T^{n m-m+1}(x)$ for each $x \in M$ and that $\left\{T^{n m-m+1}(x) \mid x \in M\right\}$ is a decomposition of $M$. The conclusion will then follow by exactly the same reasoning as in Theorem 1. Given $x \in M$ it is clear that $T^{n m-m+1}(x) \subset T^{n m-m+2}(x)$. Suppose $y \notin T^{n m-m+1}(x)$. As in the construction of Theorem 1, a sequence of continua $H_{1}, \ldots, H_{n m-m+1}$ can be constructed such that

$$
y \in H_{1}^{o} \subset H_{1} \subset H_{2}^{o} \subset H_{2} \subset \cdots \subset H_{n m-m+1}^{o} \subset H_{n m-m+1} \subset M-\{x\} .
$$

As before let $K_{i}$ be the continuum $\overline{M-H}_{i}, i=1, \ldots, n m-m+1$, and consider the pairwise disjoint open sets

$$
H_{1}^{o}, H_{2}^{o} \cap K_{1}^{o}, H_{3}^{o} \cap K_{2}^{o}, \ldots, H_{n}^{o} \cap K_{n-1}^{o}, K_{n}^{o} \text {. }
$$

Let $L_{i}$ be an irreducible subcontinuum of $M$ from $H_{i}$ to $K_{i+1}, i=1, \ldots, n m$ - $m$. Because $M$ is irreducible about $n$ points $H_{1}^{o}$ and $K_{n m-m+1}^{o}$ must each contain an end point and at least $n m-m-(n-2)$ of the $L_{i}$ 's do not contain an end point. It follows that each of these $L_{i}^{\prime}$ 's must contain $H_{i+1}^{o} \cap$ $K_{i}^{o}$ because of the irreducibility of $M$. Also because $M$ contains no simple chain of $m$ links where each link is an indecomposable continuum with nonvoid interior, at most $(m-1)(n-1)$ of the $L_{i}^{\prime}$ 's that do not contain an end point are indecomposable. Therefore at least $m n-m-(n-2)-(m$ - 1) $(n-1)=1$ of the $L_{i}$ 's does not contain an end point and is decomposable. For one such $L_{i}$ let $L_{i}=L_{a} \cup L_{b}$ where $L_{a}$ and $L_{b}$ are proper subcontinua of $L_{i}$. Since $L_{i}$ is irreducible from $H_{i}$ to $K_{i+1}$ we have $L_{a} \cap H_{i} \neq \varnothing \neq L_{b} \cap K_{i+1}$ and $L_{a} \cap K_{i+1}=\varnothing=L_{b} \cap H_{i}$. As in the proof of Theorem 1 this leads immediately to the conclusion that $T^{n m-m+2}(x)$ $\subset K_{1}$. Hence $y \notin T^{n m-m+2}(x)$ and it follows that $T^{n m-m+1}(x)=$ $T^{n m-m+2}(x)$.

To show that $\left\{T^{n m-m+1}(x) \mid x \in M\right\}$ is a decomposition of $M$, the 
reasoning in Theorem 1 may be employed with $T^{n}$ replaced by $T^{n m-m+1}$.

In Theorems 1 and 3 it is established that the structure of the elements in the decompositions are precisely $T^{n}(x)$ and $T^{n m-m+1}(x)$, respectively. It is not hard to show by means of simple examples that the exponents of $T$ cannot be reduced. Also it can be shown easily that the elements $D$ of the decomposition $\mathscr{D}$ in Theorem 2 that have void interiors are of the form $T^{n}(x)$ for each $x \in D$. But the structure of the elements with nonvoid interiors is considerably more complicated.

It might be pointed out too that, while Theorem 1 assures a decomposition space which preserves the number of "ends," the decomposition spaces of Theorems 2 and 3 may greatly reduce this number; in the case of Theorem 3, the decomposition space may be degenerate.

\section{REFERENCES}

1. H. S. Davis, D. P. Stadtlander and P. M. Swingle, Properties of the set function $T^{n}$, Portugal. Math., 21 (1962), 113-133.

2. R. W. FitzGerald and P. M. Swingle, Core decompositions of continua, Fund. Math., 61 (1967), 33-50.

3. G. R. Gordh, Jr., Monotone decompositions of irreducible Hausdorff continua, Pacific J. Math., 36 (1971), 647-658.

4. J. G. Hocking and G. S. Young, Topology, Addison-Wesley, Reading, Mass., 1961.

5. F. B. Jones, Aposyndetic continua and certain boundary problems, Amer. J. Math., 67 (1941), 545-553.

6. M. J. Russell, Monotone decompositions of continua irreducible about a finite subset, Fund. Math., 72 (1971), 255-264.

7. E. S. Thomas, Jr., Monotone decompositions of irreducible continua, Rozprawy Matematyczne, 50 (1966), 1-74.

8. E.J. Vought, Monotone decompositions of continua into generalized arcs and simple closed curves, Fund. Math., 80 (1973), 213-220.

9. L. E. Ward, Jr., Mobs, trees and fixed points, Proc. Amer. Math. Soc., 8 (1957), 798-804. 10. G. T. Whyburn, Analytic topology, Amer. Math. Soc., Colloquium Publications, 28 (1942).

Received December 28, 1972 and in revised form April 1, 1974.

California State University, Chico 



\section{PACIFIC JOURNAL OF MATHEMATICS}

\section{EDITORS}

RICHARD ARENS (Managing Editor)

University of California

Los Angeles, California, 90024

\section{R. A. BEAUMONT}

University of Washington

Seattle, Washington 98105

\section{J. DugunDJI}

Department of Mathematics University of Southern California Los Angeles, California 90007

D. Gillbarg AND J. MilgRAM

Stanford University Stanford, California 94305

\section{ASSOCIATE EDITORS}

E. F. BECKENBACH

B. H. NEUMANN

SUPPORTING

UNIVERSITY OF BRITISH COLUMBIA

UNIVERSITY OF CALIFORNIA

MONTANA STATE UNIVERSITY

UNIVERSITY OF NEVADA

NEW MEXICO STATE UNIVERSITY

OREGON STATE UNIVERSITY

UNIVERSITY OF OREGON OSAKA UNIVERSITY
F. WOLF

K. YosHIDA

INSTITUTIONS

UNIVERSITY OF SOUTHERN CALIFORNIA

STANFORD UNIVERSITY

UNIVERSITY OF TOKYO

UNIVERSITY OF UTAH

WASHINGTON STATE UNIVERSITY

UNIVERSITY OF WASHINGTON

AMERICAN MATHEMATICAL SOCIETY

NAVAL WEAPONS CENTER

The Supporting Institutions listed above contribute to the cost of publication of this Journal, but they are not owners or publishers and have no responsibility for its content or policies.

Mathematical papers intended for publication in the Pacific Journal of Mathematics should be in typed form or offset-reproduced, (not dittoed), double spaced with large margins. Underline Greek letters in red, German in green, and script in blue. The first paragraph or two must be capable of being used separately as a synopsis of the entire paper. Items of the bibliography should not be cited there unless absolutely necessary, in which case they must be identified by author and Journal, rather than by item number. Manuscripts, in duplicate if possible, may be sent to any one of the five editors. Please classify according to the scheme of Math. Rev. Index to Vol. ${ }^{39}$. All other communications to the editors should be addressed to the managing editor, or Elaine Barth, University of California, Los Angeles, California, 90024.

100 reprints are provided free for each article, only if page charges have been substantially paid. Additional copies may be obtained at cost in multiples of 50 .

The Pacific Journal of Mathematics is issued monthly as of January 1966. Regular subscription rate: $\$ 72.00$ a year (6 Vols., 12 issues). Special rate: $\$ 36.00$ a year to individual members of supporting institutions.

Subscriptions, orders for back numbers, and changes of address should be sent to Pacific Journal of Mathematics, 103 Highland Boulevard, Berkeley, California 90708.

\section{PUBLISHED BY PACIFIC JOURNAL OF MATHEMATICS, A NON-PROFIT CORPORATION}

Copyright (C) 1974 by Pacific Journal of Mathematics

Manufactured and first issued in the U.S.A. 


\section{Pacific Journal of Mathematics}

\section{Vol. 54, No. $2 \quad$ June, 1974}

John Edward Coury, Walsh series with coefficients tending monotonically to

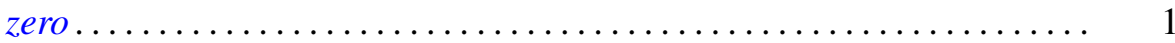

Patrick Michael Fitzpatrick and Walter Volodymyr Petryshyn, Fixed point theorems for multivalued noncompact acyclic mappings ............

Irving Leonard Glicksberg, More on Phragmén-Lindelöf for function

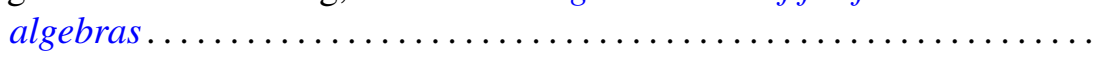

Adilson Goncalves, Structural constants. II .................. 39

Richard P. Gosselin, Closure theorems for affine transformation groups .... 53

Ralph Peter Grimaldi, Baer and UT-modules over domains ........... 59

Edward Grossman, On the prime ideal divisors of $\left(a^{n}-b^{n}\right) \ldots \ldots \ldots \ldots . \ldots 73$

A. Hedayat and Ester Seiden, On the theory and application of sum composition of Latin squares and orthogonal Latin squares.......... .

Gerald L. Itzkowitz, Continuous measures, Baire category, and uniform continuity in topological groups ......................... 115

Francis Masat, Right simple congruences on a semigroup ............ 127

Robert Harvey Oehmke, Right congruences and semisimplicity for Rees matrix semigroups..................................

Qazi Ibadur Rahman and Jan Stankiewicz, Differential inequalities and local valency . . . . . . . . . . . . . . . . . . . . . . . . . . . . . . . . . . . . . 165

William John Reed, Random points in a simplex ................ 183

Mohan S. Shrikhande, Strongly regular graphs and group divisible

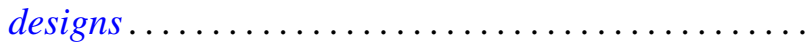

Zahava Shmuely, The structure of Galois connections ... . .

Robert C. Shock, Dual generalizations of the Artinian and Noetherian

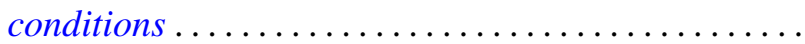

Arne Stray, Approximation and interpolation for some spaces of analytic functions in the unit disc ........................

Eldon Jon Vought, Monotone decompositions into trees of Hausdorff continua irreducible about a finite subset ............

James Wirth, The mapping cylinder axiom for WCHP fibrations ... 263

Gordon S. Woodward, Invariant means and ergodic sets in Fourier analysis... 